\title{
Statistical properties of substorms during different storm and solar cycle phases
}

\author{
N. Partamies ${ }^{1}$, L. Juusola ${ }^{1}$, E. Tanskanen ${ }^{1,2}$, and K. Kauristie ${ }^{1}$ \\ ${ }^{1}$ Finnish Meteorological Institute, Helsinki, Finland \\ ${ }^{2}$ University of Bergen, Bergen, Norway \\ Correspondence to: N. Partamies (noora.partamies@fmi.fi)
}

Received: 26 September 2012 - Revised: 28 December 2012 - Accepted: 9 February 2013 - Published: 27 February 2013

\begin{abstract}
Substorm properties during different storm phases have been studied using an automated recognition of substorm and storm phases in the auroral electrojet (AL) and ring current (Dst) index data from 1995-2009. The large number of events (about 500 storms and 15000 substorms) provides statistically reliable distributions, average behaviour and long time series of simple parameters, such as durations and intensities. The phases of storms and substorms have been examined independently. Substorm phases have been further combined to single and multi-cycle events. The former consist of one growth, one expansion and one recovery phase, while the latter include multiple expansion and recovery phases after one growth phase.

Our findings show that most substorms take place during non-storm times, and substorms during storm initial phases resemble isolated non-storm time substorms. Both during storm initial phases and non-storm times, the substorm growth phases may last longer than the other substorm phases. Substorm recovery phase is typically the longest phase but its duration also varies most. The longest substorm recovery phase duration was observed during multicycle substorms. The longest substorm expansion and storm main phases were found during the years close to the solar maximum. The shortest substorm events (the shortest phase durations) are the single-cycle substorms. The period of expansion onsets during multi-cycle substorms varied hugely for events with a small number of expansion phases. For events with a larger number of expansions, a clearer periodicity of about one hour (median value) was suggested.
\end{abstract}

Keywords. Magnetospheric physics (Magnetosphereionosphere interactions; Solar wind-magnetosphere interactions; Storms and substorms)

\section{Introduction}

Substorms have been recognised as an important mechanism for transferring and dissipating the magnetospheric excess energy to the ionosphere and regions close to the Earth. They have been intensively studied during the past years and decades, mainly in terms of event studies. The ideal case of a single substorm during a magnetically quiet period as a starting point, effort has also been put into understanding substorms during magnetic storms. This more complex scenario during large-scale disturbances has not yet been fully characterised.

The typical length of the substorm event is about $2-4 \mathrm{~h}$. It includes at least one growth, expansion and recovery phase (e.g. McPherron, 1970, 1979), whose durations vary from the short expansion of 15-20 min, to a lengthy growth or recovery of a few hours (e.g. Kallio et al., 2000; Tanskanen, 2009; Gjerloev et al., 2007). Similarly, durations of 1.3-2.3 h for the combination of expansion and recovery phases were found by Kullen and Karlsson (2004) in a survey of global auroral images from the Polar spacecraft. The growth phase cannot be reliably determined from the global images, so it was excluded in their study. The average duration of substorms observed in 1993-2003 by Tanskanen (2009) is about $3 \mathrm{~h}$, while the full range of the yearly averages varied from 2.8 to $3.3 \mathrm{~h}$. Tanskanen (2009) defined the onsets from the decrease of a local electrojet (IL) index (Kallio et al., 2000). The decrease was required to be at least $100 \mathrm{nT}$ and the rate of decrease was more than $80 \mathrm{nT}$ in $15 \mathrm{~min}$. The start of the substorm growth was defined as the time when the IL index showed the first signs of a negative bay, but not more than $30 \mathrm{~min}$ before the onset time. The substorm was concluded to be over when the IL index had recovered $80 \%$ of its peak 
deflection. In 1997, the average substorm duration was 15 $20 \mathrm{~min}$ shorter than the mean value of $3 \mathrm{~h}$ for all 11 years. The length of the substorm period also increased slightly towards the year 2003. The average substorm duration in 2003 represents the average duration of substorms during active years after the solar maximum.

The repetitive substorms are often related to magnetic storms. A typical cadence of $2-3 \mathrm{~h}$ has been reported as the substorm recurrence rate (e.g. Borovsky et al., 1993; Pulkkinen et al., 2007). Magnetic storms are longer term geomagnetic activity periods for which the Dst index reaches a minimum of at least $-40 \mathrm{nT}$ (Kallio et al., 2000). An early storm model by Gonzalez et al. (1994) examines the ring current enhancement, as measured by the Dst index, with a superposition of a number of substorm disturbances. This simple formulation results in a conclusion that, apart from the most intense and frequent substorms taking place during storm main phases (the interval of the largest decrease of the Dst index), there are no apparent differences between quiet time and storm-time substorms. Similar conclusions were later brought up by e.g. McPherron and Hsu (2002). The storm initial phase is often believed to start with a solar wind trigger, which for intense storms tends to be an interplanetary shock front. The storm main phase is typically accompanied by sustained southward interplanetary magnetic field (IMF). The storm recovery phase lasts until the magnetosphere has returned to nominal conditions and most of the excess energy delivered by the solar wind has been dissipated.

A recent case study of substorms during different storm phases (Partamies et al., 2011) suggested that the substorms prior to the storm main phase behave as isolated substorms, while the storm main phase substorms show an extremely intense nature due to the enormous amount of energy provided by the solar wind. The most peculiar substorm characteristics were found during the recovery phase of the magnetic storm, where the substorm activity was long-lasting but moderate in intensity. These findings are based on a case study of a weak magnetic storm. Therefore, there is a need for a statistical study of substorms during magnetic storms to resolve the average behaviour of storm-time substorms in addition to single event samples. The statistical approach requires an automatic identification of substorms, magnetic storms and their phases. An automatic substorm identification was recently published by Juusola et al. (2011). They used a simple routine to automatically determine substorm phases in the auroral electrojet (AE) index data. We adopt their definitions and routines for finding substorm phases and follow similar logic for searching magnetic storms and storm phases in the Dst index data.

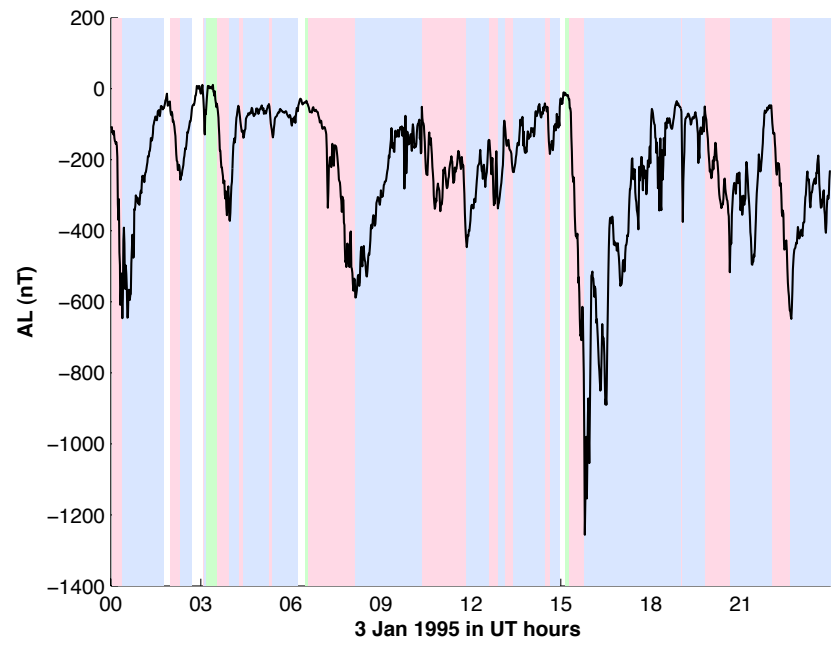

Fig. 1. An example of substorm phases identified from AL index: growth phase in light green, expansion phase in pink and recovery phase in light blue shading.

\section{Event identification}

\subsection{Substorm phases}

According to Juusola et al. (2011), we use the solar wind IMF data from OMNI Web, and the AE index data, in particular the lower (AL) envelope curve, for a time period of 15 years in 1995-2009 for identifying substorm events and their growth, expansion and recovery phases. The search criteria are:

1. Growth phase: from IMF $B_{\mathrm{Z}}$ southward turn until the substorm onset;

2. Substorm onset: abrupt decrease of $\mathrm{AL}, \mathrm{dAL} / \mathrm{d} t<$ $-4 \mathrm{nT} \min ^{-1}$, with a minimum $\mathrm{AL}$ value less than $-50 \mathrm{nT}$;

3. Expansion phase: from the substorm onset until the local AL index minimum;

4. Recovery phase: from the $\mathrm{AL}$ index minimum until $\mathrm{AL}$ has reached $-50 \mathrm{nT}$ or a new onset.

The minimum AL value of $-50 \mathrm{nT}$ is the median of all negative AL values over the full set of data. With these criteria, the 15-year period included 15568 growth, 54519 expansion and 53551 recovery phases. This sums up to the total durations of 524 days (1.4 years) of growth phase, 896 days ( 2.5 years) of expansion and 1787 days (5 years) of substorm recovery phase. More detailed description of the phase recognition algorithm and the trimming of the phases into continuous periods can be found in Juusola et al. (2011). Figure 1 illustrates how substorm phases are seen in the AL index on 3 January 1995. Phases identified by the routine described above have been shaded in light green, pink and light blue 
for substorm growth, expansion and recovery phases, respectively. This day is an example of a very active day when there are only a few short time periods of quiet time (white), where quiet is the time without another substorm phase label. Each substorm during this day includes several expansionrecovery phase cycles. The day consists of 3 growth phases, 16 expansion and recovery phases as well as 4 quiet time periods, whose total durations are $38 \mathrm{~min}$ (growth), $7.3 \mathrm{~h}$ (expansion), $15.2 \mathrm{~h}$ (recovery) and $59 \mathrm{~min}$ (quiet), respectively.

\subsection{Storm phases}

Following the substorm selection procedure by Juusola et al. (2011), we further define storm phases from the 15-years of Dst index data:

1. Initial phase: Dst $>0$;

2. Main phase: abrupt decrease of Dst, $\mathrm{dDst} / \mathrm{d} t<$ $-2 \mathrm{nT} \mathrm{h}^{-1}$, with a minimum Dst value less than $-15 \mathrm{nT}$;

3. Recovery phase: from the Dst minimum until Dst has returned to values larger than $-15 \mathrm{nT}$.

Similarly to the substorm phase trimming described by Juusola et al. (2011), the shortest storm phases have been removed and overlapping phases given the following priority: (1) main phase, (2) recovery phase and (3) initial phase.

The minimum required Dst value of $-15 \mathrm{nT}$ is the median of all negative Dst values over the full length of data. With these criteria, the whole Dst data set consists of 480 initial, 1911 main and 1862 storm recovery phases, whose total durations are 492 days ( 1.3 years) of initial, 450 days ( 1.2 years) of main and 1654 days ( 4.5 years) of storm recovery. The logic of the storm phase identification closely follows that of the substorm phase identification. An example storm as Dst index evolution is plotted in Fig. 2. The automatically identified storm phases have been marked by light green, pink and light blue shading for initial, main and recovery phase, respectively. White sections correspond to non-storm times.

We use Dst index rather than the pressure-corrected Dst index (Gonzalez et al., 1989) for easier availability. The solar wind pressure correction has the largest effect on Dst during intense storms, during which the Dst index minimises below $-100 \mathrm{nT}$. These events comprise only about $1 \%$ of the storms identified by our search routine.

\section{Results}

\subsection{Median values of the entire data set}

For most of the analysis in this study, the phases of substorms and magnetic storms are examined separately without grouping phases into substorm or storm events. The median durations of the substorm phases were $31 \mathrm{~min}$ for the growth, $12 \mathrm{~min}$ for the expansion and $31 \mathrm{~min}$ for the recovery,



Fig. 2. An example of storm phases identified from Dst index: Initial phase in light green, main phase in pink and recovery phase in light blue shading.

Table 1. Activity index median values during all substorm phases. The last row gives the mean duration values for the different phases.

\begin{tabular}{lcccc}
\hline & Growth & Expansion & Recovery & Quiet \\
\hline AE (nT) & 70 & 187 & 186 & 61 \\
AU (nT) & 41 & 69 & 69 & 35 \\
AL (nT) & -29 & -111 & -110 & -24 \\
Dst (nT) & -8 & -17 & -16 & -8 \\
Duration (min) & 31 & 12 & 31 & 75 \\
Avg. duration (min) & 48 & 24 & 48 & 200 \\
\hline
\end{tabular}

but their modes were much shorter: 14 min for the growth, $14 \mathrm{~min}$ for the expansion, and $10 \mathrm{~min}$ for the recovery. The median values are comparable with the previous substorm studies. The median values of the magnetic activity indices for each substorm phase are given in Table 1. The table values clearly show that typically, the expansion and recovery phase are identical in intensity but the expansion phase is much shorter in duration. Due to the skew of the phase duration distribution, the last row of Table 1 shows the mean values of the substorm phase lifetimes.

Similarly, we present the median index values and durations for all of the storm phases in Table 2. For storm phases, the differences between the main and the recovery phases are larger than the differences between the substorm expansion and recovery. This is a consequence of the larger difference of storm phase lifetimes as compared to the substorm phase lifetimes. With the longer lifetimes of storm recovery phases, a different distribution of index values becomes more likely. The positive Dst value during the storm initial phase reflects the selection criterion. Similarly to the storm phase index value, the last row of Table 2 lists the average durations of 
Table 2. Median values for activity indices and durations during all storm phases. The last row shows the mean duration values for the different phases.

\begin{tabular}{lcccc}
\hline & Initial & Main & Recovery & Non-storm \\
\hline AE (nT) & 71 & 361 & 218 & 76 \\
AU (nT) & 39 & 119 & 80 & 37 \\
AL (nT) & -26 & -229 & -127 & -34 \\
Dst (nT) & 4 & -23 & -24 & -8 \\
Duration (h) & 13 & 4 & 16 & 36 \\
Av. duration (h) & 25 & 6 & 21 & 69 \\
\hline
\end{tabular}

the substorm phases to give the reader an idea of the asymmetry of the lifetime distributions.

\subsection{Solar cycle dependence of storm and substorm phases}

Figure 3 shows the numbers of the storm main phases and non-storm times per year (top panel) and the numbers of the substorm expansion phases and quiet times per year (bottom panel). The yearly numbers of main and expansion phases are marked by solid lines, and the yearly numbers of nonstorm and quite times by blue asterisks. The solar minima in 1996-1997 and 2008-2009 (blue dotted lines) can be seen as a lack of magnetic activity in both storms and substorms, while the years 2002-2003 after the solar maximum (red dotted line) showed an increase in the number of storms. The substorm occurrence rate appears very stable apart from the deepest years of the solar minima. The quiet and non-storm time numbers are small compared to the numbers of the active periods, except during the latest and exceptionally deep solar minimum, which resulted in very few magnetic storms in 2006-2009. The yearly median durations of storm main phases and substorm expansion phases are marked by black numbers above the solid black curves, main phase durations in hours and expansion phase durations in minutes, respectively. The yearly median durations of non-storm periods (in days) and quiet times (in hours) are shown by the blue numbers below each of the blue asterisks. The figure has no axis for the median duration values. In particular, the median duration of non-storm and quiet times (blue numbers) reaches maximum in 2009 ( 8 days for non-storm times and $2.6 \mathrm{~h}$ for quiet periods), while the median lengths of storm main phases and substorm expansion phases (black numbers) maximise in 2003 ( $5 \mathrm{~h}$ for main and $16 \mathrm{~min}$ for expansion phases). The median duration of storm main phases is not much affected by the solar cycle but the shortest substorm expansion phases are observed during the deep minimum in 2009.

The annual distribution of the total duration of different storm and substorm phases is shown in Fig. 4. Initial and growth phases have been plotted by green, main and expansion phases as red, recovery phases as blue and non-storm and quiet times as black curves. Here the solar minima (1997
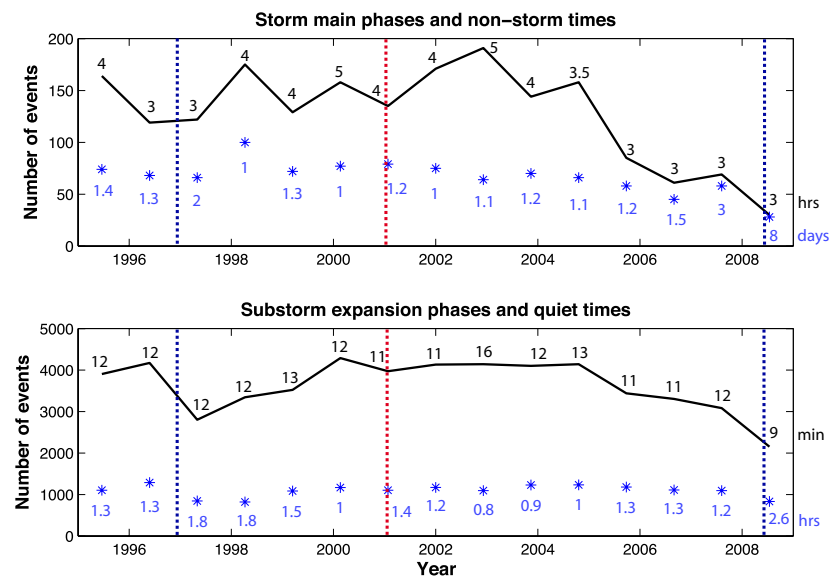

Fig. 3. Annual distribution of storm main phases and non-storm times (top) and substorm expansion phases and quiet times (bottom). The numbers of non-storm (top panel) and quiet times (bottom panel) are marked with blue asterisks. Annual median duration values are given for main phases (top panel) in hours and expansion phases (bottom panel) in minutes, both in black numbers. Correspondingly, annual median durations of quiet times (bottom panel) are annotated in minutes and non-storm times (top panel) in days, both in blue numbers. The vertical dotted lines mark the solar minimum years (blue) and solar maximum year (red).

and 2008) can be recognised as years with very high duration of non-storm and quiet times. During the years after the solar maximum (2002-2003), the total duration of storm and substorm recovery phases (blue) exceeds the values for any other years and the time of non-storm and quiet times (black) minimises. The total durations of initial, growth, main and expansion phases (green and red) are much shorter as compared to the durations of recovery and less active times (blue and black). Also in this figure, the exceptionality of the minimum of the solar cycle 23 can be seen in 2006-2009.

\subsection{Storm initial phase substorms}

The median values for substorm phases during storm initial phase are listed in Table 3. The numbers are very similar to those averaging the entire data set of substorm phases, except that the Dst values are positive and AL index values are less negative during the storm initial phase than for all substorms. Duration of substorm expansion and recovery phases are shorter during the storm initial phase as compared to the median values of the entire data set. Quiet periods occupy most of the storm initial phases and their median is longer than the full data set values. The bottom row in the table gives the percentage of each substorm phase out of the total duration of all storm initial phases (492 days). While there is only $10 \%$ of growth phases and $8 \%$ of expansion phases, the quiet time occurs during $43 \%$ of the storm initial phases (bottom row). Altogether, $77 \%$ of storm initial phase substorms are included in the table. The substorm phase occurring partly 

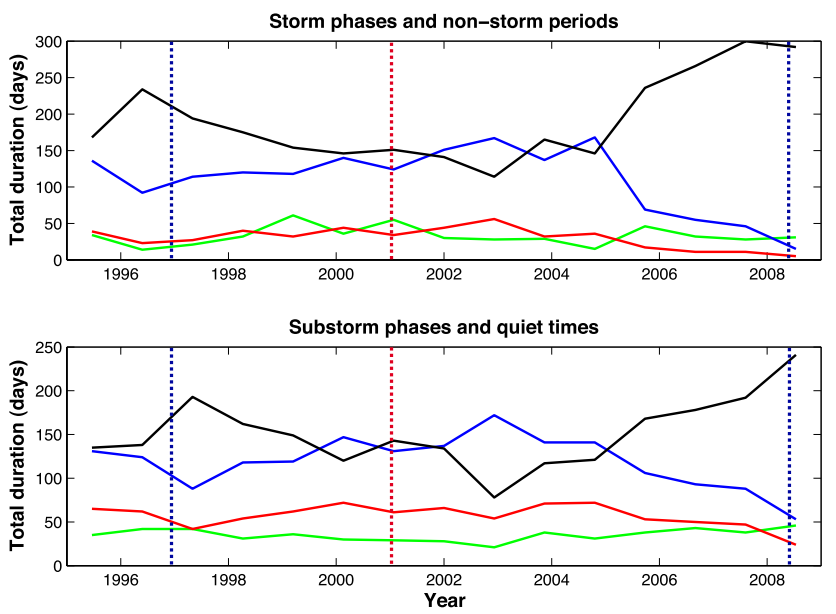

Fig. 4. Annual distribution of total duration of storm phases and non-storm times (top) and substorm phases and quiet times (bottom). Initial and growth phases are green, main and expansion phases red, recovery phases blue and non-storm and quiet times black. The vertical dotted lines mark the solar minimum years (blue) and solar maximum year (red).

Table 3. Median values of substorm phases during storm initial phases.

\begin{tabular}{lcccc}
\hline & Growth & Expansion & Recovery & Quiet \\
\hline AE (nT) & 71 & 155 & 157 & 64 \\
AU (nT) & 43 & 63 & 64 & 38 \\
AL (nT) & -27 & -84 & -87 & -24 \\
Dst (nT) & 5 & 3 & 3 & 7 \\
Median duration (min) & 33 & 9 & 26 & 88 \\
Total duration (days) & 48 & 39 & 81 & 211 \\
\% of the storm phase & 10 & 8 & 16 & 43 \\
\hline
\end{tabular}

within and partly outside the storm initial phases have been excluded for clarity.

\subsection{Storm main phase substorms}

Storm main phase substorm properties in Table 4 clearly describe the most intense set of substorms. All index values for substorm expansion and recovery phases are approximately double compared to those of the full event set. Substorm growth phases and the quiet time periods are shorter than average during the storm main phases, but they are also the minority (only $6 \%$ together) of the substorm phases during this storm phase. This suggests that a single substorm growth phase during a storm main phase is generally followed by a long cycle of repetitive substorm expansion and recovery phases, i.e. substorm intensifications. Substorm expansion and recovery phases are typically long-lasting during the storm main phases, and consequently, they occupy $63 \%$ of all storm main phase time. The table includes $69 \%$ of substorms identified during storm main phases. This num-
Table 4. Median values of substorm phases during storm main phases.

\begin{tabular}{lcccc}
\hline & Growth & Expansion & Recovery & Quiet \\
\hline AE (nT) & 86 & 344 & 346 & 88 \\
AU (nT) & 58 & 124 & 131 & 59 \\
AL (nT) & -29 & -208 & -205 & -28 \\
Dst (nT) & -18 & -26 & -28 & -21 \\
Median duration (min) & 23 & 23 & 34 & 29 \\
Total duration (days) & 15 & 125 & 156 & 15 \\
$\%$ of the storm phase & 3 & 28 & 35 & 3 \\
\hline
\end{tabular}

Table 5. Median values of substorm phases during storm recovery phases.

\begin{tabular}{lcccc}
\hline & Growth & Expansion & Recovery & Quiet \\
\hline AE (nT) & 80 & 221 & 218 & 69 \\
AU (nT) & 47 & 82 & 80 & 42 \\
AL (nT) & -32 & -132 & -130 & -27 \\
Dst (nT) & -26 & -27 & -27 & -22 \\
Median duration (min) & 23 & 13 & 33 & 46 \\
Total duration (days) & 105 & 343 & 701 & 289 \\
\% of the storm phase & 6 & 21 & 42 & 17 \\
\hline
\end{tabular}

ber is smaller than for other storm phases simply because the storm main phases have the shortest average duration, making it more likely that any associated substorm phase would not occur completely inside the storm phase.

\subsection{Storm recovery phase substorms}

The typical substorm phase values during storm recovery phases are listed in Table 5. The substorm expansion and recovery, again, have a very similar nature in the strength. However, the substorm expansion phases last for much shorter periods. The total duration of recovery phases is clearly the most dominant. This is in agreement with the recent case study and emphasizes the importance of substorm recovery phases in dissipating the excess energy at the end of magnetic storms (Partamies et al., 2011). Substorm growth phase durations are identical to the growth phase durations observed during storm main phases, but the growth phases comprise the smallest fraction of all storm recovery phases. The substorm phases that begin and end within a storm recovery phase include $86 \%$ of all identified substorm phases in duration.

\subsection{Non-storm time substorms}

As a reference for the storm-time substorms, we also include the median values of the substorm phases during the nonstorm times (Table 6). In this case, the index values are comparable to the full set of substorms suggesting that most substorms occur during non-storm times. Apart from the slightly negative Dst, the index values are similar to those during 
Table 6. Median values of substorm phases during non-storm times.

\begin{tabular}{lcccc}
\hline & Growth & Expansion & Recovery & Quiet \\
\hline $\mathrm{AE}(\mathrm{nT})$ & 65 & 151 & 152 & -56 \\
$\mathrm{AU}(\mathrm{nT})$ & 36 & 53 & 53 & 31 \\
$\mathrm{AL}(\mathrm{nT})$ & -28 & -91 & -92 & -23 \\
Dst (nT) & -5 & -8 & -8 & -5 \\
Median duration (min) & 36 & 9 & 28 & 88 \\
Total duration (days) & 331 & 303 & 380 & 1360 \\
$\%$ of the storm phase & 11 & 11 & 13 & 47 \\
\hline
\end{tabular}

storm initial phases (Table 3). The characteristic substorm phase durations are also in agreement with the corresponding durations during storm initial phases.

\subsection{Substorm cycle structures}

We further tracked the structure of the substorm phases by searching for a follow-up phase for each individual substorm growth phase. By definition, each growth phase is followed by an expansion phase, and each expansion phase is followed by a recovery phase. But after a recovery phase there can be another expansion and recovery phase and the number of repetitions of these is indeterminate. Events excluded from this examination either had no growth phase or ended in an expansion.

In the case of 7181 substorms $(\sim 46 \%$ of all $\sim 15000$ substorms), the phase cycle goes from growth to expansion and recovery, and ends there. These "textbook" substorm events occur throughout the whole data period (top panel of Fig. 5) being more common during solar minimum years (1996, 2006-2009). They are accompanied by index values that are about two thirds of the corresponding values for the full set of substorms (Table 1). The median durations for single-cycle substorm expansions and recovery phases are short (8 and $19 \mathrm{~min}$, respectively) compared to the average of the full set or any storm phase. As another extreme, 5124 substorms $(\sim 32 \%$ of all substorms and including a total of 23291 expansion phases) consist of two or more expansionrecovery cycles after the growth phase. The median auroral electrojet (AE/AL/AU) index values for this event set are $\sim 10 \%$ higher as compared to the full event set (Table 1). The Dst median values and median phase durations are identical. Compared to the single-cycle substorms, the median index values during the multi-cycle events double.

Both the single- and multi-cycle substorms are present throughout the 15-year time period and even somewhat similarly distributed, as is shown in Fig. 5.

The multi-cycle substorm occurrence follows slightly closer the variations in the daily sunspot number (red curve in the figure) showing minimum values in 1997-1998 and in 2009. A peculiar maximum for both event groups can be seen in 1996. Single-cycle substorms were clearly more common during the last extreme solar minimum in 2006-2009. Fur-
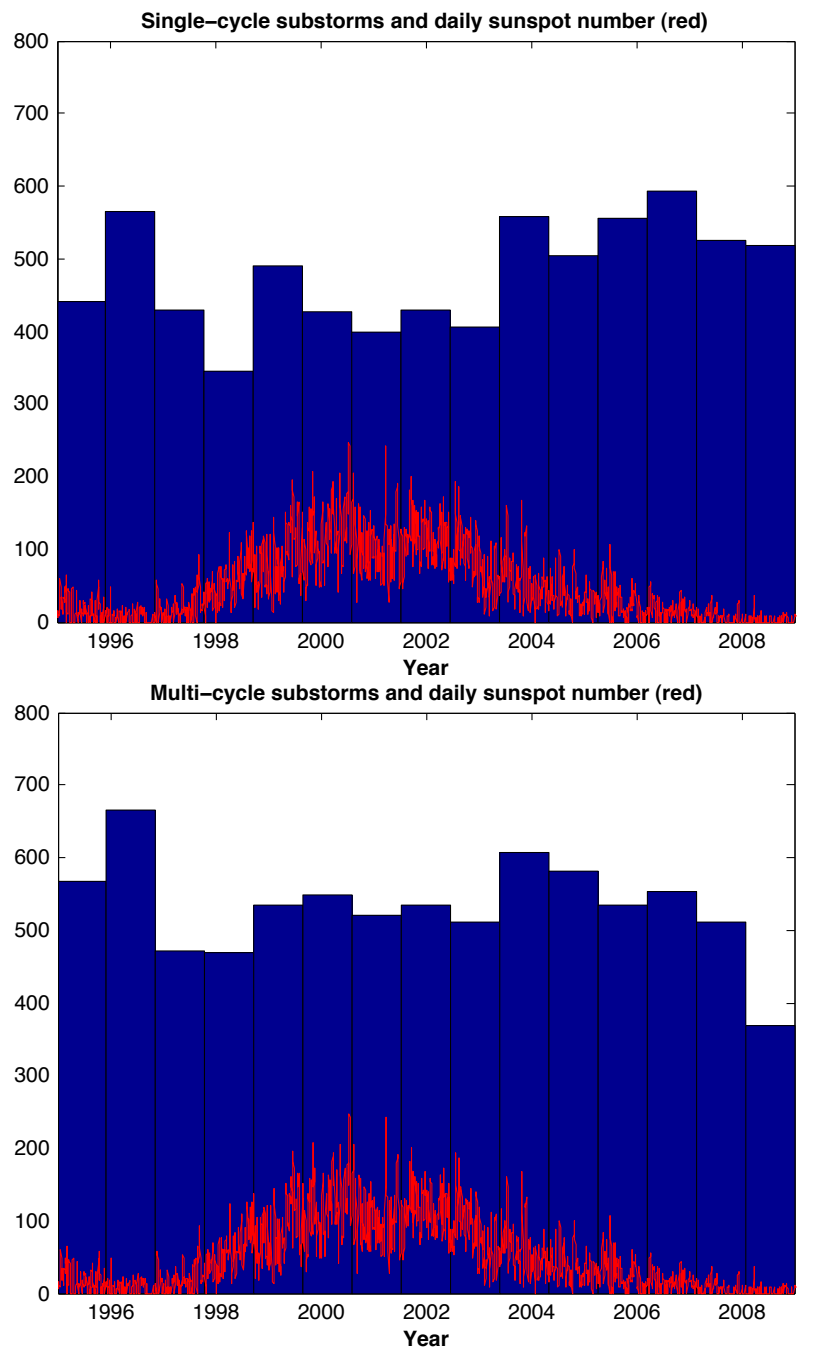

Fig. 5. Annual numbers of single-cycle substorms (top panel blue bars) and multi-cycle substorm events (bottom panel blue bars) together with the daily sunspot number (red curve).

thermore, the substorms during the past deep minimum were dominated by less intense events (AL typically $\sim-80 \mathrm{nT}$ ), while during the most active years the event set consisted of a significant number of more intense substorms (AL typically $\sim-140 \mathrm{nT})$.

In total about $37 \%$ of the single-cycle substorms and about $46 \%$ of multi-cycle substorms take place during magnetic storms (initial, main or recovery phase). Comparing durations of the single and multi-cycle substorms phases during magnetic storm phases, we found that the recovery phase lengths vary the most. The values for median and total durations of single-cycle and multi-cycle substorm recovery phases for different states of storms are listed in Table 7.

Single-cycle substorms during storms generally recover much faster than multi-cycle events. Both event groups are clearly most numerous during the non-storm time but the multi-cycle substorms occupy a fair duration during the 
Table 7. Median values of substorm recovery phase durations of single and multi-cycle substorms.

\begin{tabular}{lcccc}
\hline & Initial & Main & Recovery & Non-storm \\
\hline Single-cycle events & & & & \\
\hline Median duration (min) & 19 & 11 & 17 & 20 \\
Total duration (days) & 15 & 4 & 40 & 96 \\
\hline Multi-cycle events & & & & \\
\hline Median duration (min) & 32 & 35 & 37 & 33 \\
Total duration (days) & 15 & 24 & 8 & 121 \\
\hline
\end{tabular}

storm main phases, while single-cycle substorms are very few during the storm main phases but clearly more common during storm recovery phases.

The set of multi-cycle substorms allowed us to study the periodicity of substorm expansion phases (onsets) for each event. The relation between the period and the number of expansions is illustrated in Fig. 6. Each asterisk in the figure corresponds to the median period within one multi-cycle substorm event and the number of expansions during that event. The median value of the substorm expansion periods (red curve) is slightly less than one hour for the events including 2-5 expansion onsets, but increases by about $20 \mathrm{~min}$ (period of $\sim 1.3 \mathrm{~h}$ ) for the longest substorm cycles. Although the deviation in the onset periods between different events is much larger for events with small numbers of expansions than for longer substorm cycles, the median and quartile values (red and green curves) remain rather constant.

\section{Discussion}

The substorm phases examined in this study mainly follow the definitions of earlier substorm studies. The expansion phase lasting for the lifetime of the fast decrease of AL index (also used by Juusola et al., 2011) results in shorter substorm expansion phases than what have been reported in some of the previous substorm studies (e.g. Chua et al., 2004; Hoffman et al., 2010). Furthermore, our substorm phase identification routine has a median AL value $(-50 \mathrm{nT})$ as a threshold for the end of a substorm instead of threshold values that are related to the minimum AL of each event. For instance, recovery of $80 \%$ of the minimum value has determined the end of substorm events analysed by Partamies et al. (2011); Tanskanen (2009). This makes the selection criteria used in this paper more sensitive for new substorm expansion phases in the middle of recovery phases. These are good things to keep in mind when comparing our results with earlier statistics.

The typical substorm phase durations found in this study were $31 \mathrm{~min}$ for the growth phase, $12 \mathrm{~min}$ for the expansion phase and $31 \mathrm{~min}$ for the recovery phase. These values sum up to an agreement $(74 \mathrm{~min})$ with the early substorm studies by Rostoker et al. (1987, 1980), who suggested

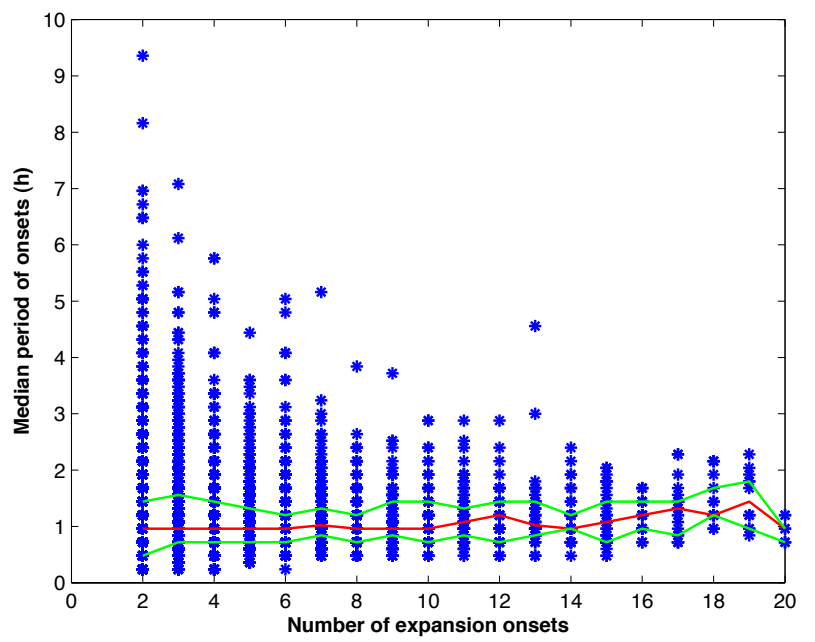

Fig. 6. Dependence of the period of consecutive substorm expansion onsets on the number of expansions in the event. Median periods are marked by the red line and $25 \%$ and $75 \%$ quartiles by green curves.

that the lifetime of the full substorm event is $1-3 \mathrm{~h}$. However, during storm-times the median duration of our substorm events appear even about $10 \mathrm{~min}$ shorter. A more recent study by Jayachandran and MacDougall (2007) examined 26 substorms whose average expansion phase duration (determined from the polar cap convection measurements) was $22.4 \mathrm{~min}$, while geosynchronous orbit magnetic field measurements suggested an average expansion phase duration of $5.8 \mathrm{~min}$.

The threshold value for substorms ( $\mathrm{AL}<-50 \mathrm{nT})$ is constant in our study. However, the ionospheric conductivity is higher and electrojet indices reach higher values during the summer months. Unlike for identification of steady magnetospheric convection events (Partamies et al., 2009), where this causes an artificial occurrence maximum, it probably results in an unrealistically even monthly distribution of substorms. Adjusting the AL index threshold value according to the statistical variation of it from month to month should be further tested in the future.

The median index values of the substorm phases are moderate or small as compared to the previous studies by e.g. Tanskanen et al. (2002). This is due to the low threshold value of $\mathrm{AL}$ and its constant nature. A more typical threshold value for the substorm intensity in automated identification is $100 \mathrm{nT}$, as was used in a local electrojet index study by Tanskanen et al. (2005). Worth noticing, however, is the fact that a local electrojet index has also been used in the study by Tanskanen et al. (2002) instead of the global AL index. For events which take place right in the time sector of interest, the local index records larger magnetic deflections.

According to our study, during non-storm conditions a lengthy substorm growth phase leads to a short expansion 
and recovery phase, where the input energy is efficiently dissipated and the magnetosphere returns to quiet.

Tanskanen (2009) showed that the substorm duration increases during the declining phase of the solar cycle (towards 2003). In our event set the expansion phases shorten towards 2003 (Fig. 3). The shorter expansion phases are compensated by the substorm recovery phases becoming more dominant in terms of their total duration during the years following the sunspot maximum.

According to our data set (Fig. 6), the median period of the multi-cycle substorms is rather independent on the number of expansion and recovery phases during the event. The median value varies only slightly around one hour, which is much shorter than the typical recurrence rate of consecutive substorms of 2-3 h (Pulkkinen et al., 2007; Borovsky et al., 1993). Our multi-cycle substorm period is also short compared to the periods found during sawtooth events. Cai and Clauer (2009) reported a mean value for sawtooth periods of about $3 \mathrm{~h}$, with very few events with periods of the order of one hour. Periods of four sawtooth events analysed by Kubyshkina et al. (2008) varied considerably within one event $(0.5-3.5 \mathrm{~h})$. Their study showed a linear relationship between the period and the driving solar wind electric field for those few sample events. Thus, in terms of periodicity, the multi-cycle substorms have a shorter waiting time and less logical ordering.

We found no systematic onset period changes within substorm events, but the lengthening and shortening of the period within a single event seemed random. Typically the period varied by some tens of minutes during one event. The longest periods and cycles are at the limit of meaningfulness of the identification routine. The longest periods (longer than about $6 \mathrm{~h}$ ) are events which a trained eye would split into several expansions and recoveries although the search criteria are just about not fulfilled. Respectively, the longest cycles take place during disturbed periods when the criterion for a new expansion is met more easily than what a trained observer would suggest. In these conditions, a full recovery is also challenging to find. Note that the disturbances do not need to be very intense but milder fluctuations in the index data are enough to keep the substorm cycle evolution going.

The variations in the durations of the substorm phases are large and highly dependent on the grouping of events according to the storm-activity. The shortest and longest median durations for substorm growth, expansion and recovery phase found in this study are 23 and $36 \mathrm{~min}, 9$ and $23 \mathrm{~min}, 11$ and $37 \mathrm{~min}$, respectively. In this light, it is not surprising that the case studies found in the literature are so different from each other. In fact, the substorm phase lifetimes during different storm phases reported by Partamies et al. (2011) are much longer than the statistical values found in this study. The largest range of lifetimes is covered by the durations of substorm recovery phases, from $11 \mathrm{~min}$ of the single-cycle substorm recoveries during the storm main phases to the $37 \mathrm{~min}$ of the multi-cycle substorm recoveries during the storm re- covery phases (Table 7). In addition, the substorm recovery phases occupy most of the total duration of all substorms. Considering the median index values, the substorm recovery phase is typically as intense as the expansion phase, and thus plays an important role in dissipating the storm energy, even when the role of the expansion phase is emphasised during storm-times. This is in agreement with the results of an event study by Partamies et al. (2011). Yet, there are a lot of unanswered questions about substorm recovery phase processes.

This study has not considered the different modes of the magnetosphere, i.e. sawtooth events (e.g. Herderson et al., 2006) and steady magnetospheric convections (SMC, Sergeev et al., 1996; DeJong and Clauer, 2005). Our analysis is based on automatic event identification in the AL index data where the loading-unloading processes (quiet-time and storm-time substorms as well as sawtooth events) look alike. A distinguished magnetospheric mode is SMC, which typically begins with a substorm and resembles a prolonged recovery phase of the substorm. Without visually inspecting and manually excluding these SMC related substorms we have introduced a bias towards lengthy substorm recovery phases. However, the annual number of SMC events in the previous studies (e.g. Partamies et al., 2009; O'Brien et al., 2002) has been of the order of hundred events, while the current statistics include over 3000 substorm recovery phases per year. Thus, we conclude that the bias of SMC recovery phases is marginal. Furthermore, the substorm expansion phase onsets used in this study has been compared to the list of onset identified from the IMAGE satellite data (Frey et al., 2006). As has been demonstrated by Juusola et al. (2011), these two onset times are in good agreement. Juusola et al. (2011) further showed that the substorms studied here are associated with earthward flows in the magnetotail.

\section{Summary and conclusions}

In this study, we have analysed 15 years of auroral electrojet and Dst index data from 1995-2009. An automatic selection routine identified 15568 growth, 54519 expansion and 53551 recovery phases of substorms, and 480 initial, 1911 main and 1862 recovery phases of magnetic storms. Although there is no ground truth for the substorm and storm phase identification, these event numbers are statistically meaningful and complement the large number of event studies of storms and substorms found in the literature.

Our main findings can be summarised as:

- The majority of substorms occurs during non-storm times.

- In terms of intensity, substorms during non-storm times and storm initial phases are very similar.

- Median intensity values of substorm expansion and recovery phases are comparable. 
- During storm initial phases and non-storm times the substorm growth typically goes on for longer than other substorm phases. Otherwise, the substorm recovery phase is the most dominant phase in terms of its total duration.

- Both storms and substorms loosely follow the sunspot activity cycle with a 1-2 year-time lag. The longest median durations of expansion and main phases were found during years close to solar minima.

- The shortest substorms are the single-cycle substorms which are only comprised of one growth, expansion and recovery phase.

- The occurrence of multi-cycle substorms is slightly more likely during storm-times than the occurrence of the single-cycle substorm events. Multi-cycle substorms have the longest substorm recovery phases.

- Very different onset period values (time separations of consecutive expansion onsets) were found during multi-cycle substorm events with only a few expansion phases. The spread decreases as the number of expansion phases increases. The median period value depends very little on the number of expansion onsets and has a value of about one hour.

Acknowledgements. The authors want to thank S.-A. Grönroos for fruitful discussions. AE and Dst index data are downloaded from the World Data Center for Geomagnetism in Kyoto at http://wdc. kugi.kyoto-u.ac.jp. Solar wind and interplanetary magnetic field data were downloaded from OMNI Web at http://omniweb.gsfc. nasa.gov/.

The authors acknowledge the financial support by the Academy of Finland, projects 115947, 128189, 128632, 108518 and 137900.

Topical Editor R. Nakamura thanks X. Cai and one anonymous referee for their help in evaluating this paper.

\section{References}

Borovsky, J. E., Nemzek, R. J., and Belian, R. D.: The occurrence rate of magnetospheric-substorm onsets: random and periodic substorms, J. Geophys. Res., 98, 3807-3813, 1993.

Cai, X. and Clauer, C. R.: Investigation of the period of sawtooth events, J. Geophys. Res., 114, A06201, doi:10.1029/2008JA013764, 2009.

Chua, D., Parks, G., Brittnacher, M., Germany, G., and Spann, J.: Auroral substorm timescales: IMF and seasonal variations, J. Geophys. Res., 109, A03207, doi:10.1029/2003JA009951, 2004.

DeJong, A. D. and Clauer, C. R.: Polar UVI images to study steady magnetospheric convection events: Initial results, Geophys. Res. Lett., 32, L24101, doi:10.1029/2005GL024498, 2005.

Frey, H. U., Mende, S. B., Angelopoulos, V., and Donovan, E. F.: Substorm onset observations by IMAGE?FUV, J. Geophys. Res., 109, A10304, doi:10.1029/2004JA010607, 2004.
Gjerloev, J. W., Hoffman, R. A., Sigwarth, J. B., and Frank L. A.: Statistical description of the bulge-type auroral substorm in the far ultraviolent, J. Geophys. Res., 112, A07213, doi:10.1029/2006JA012189, 2007.

Gonzalez, W. D., Tsurutani, B. T., Gonzalez, A. L. C., Smith, E. J., Tang, F., and Akasofu, S.-I.: Solar wind-magnetosphere coupling during intense magnetic storms (1978-1979), J. Geophys. Res., 94, 8835-8851, 1989.

Gonzalez, W. D., Joselyn, J. A., Kamide, Y., Kroehl, H. W., Rostoker, G., Tsurutani, B. T., and Vasyliunas, V. M.: What is a geomagnetic storm?, J. Geophys. Res., 99, 5771-5792, 1994.

Henderson, M. G., Reeves, G. D., Skoug, R., Thomsen, M. F., Denton, M. H., Mende, S. B., Immel, T. J., Brandt, P. C., and Singer, H. J.: Magnetospheric and auroral activity during the 18 April 2002 sawtooth event, J. Geophys. Res., 111, A01S90, doi:10.1029/2005JA011111, 2006.

Hoffman, R. A., Gjerloev, J. W., Frank, L. A., and Sigwarth, J. W.: Are there optical differences between storm-time substorms and isolated substorms?, Ann. Geophys., 28, 1183-1198, doi:10.5194/angeo-28-1183-2010, 2010.

Jayachandran, P. T. and MacDougall, J. W.: Substorm time scales from polar cap convection measurements, Earth Planets Space, 59, e29-e32, 2007.

Juusola, L., Østgaard, N., Tanskanen, E., Partamies, N., and Snekvik, K.: Earthward plasma sheet flows during substorm phases, J. Geophys. Res., 116, A10228, doi:10.1029/2011JA016852, 2011.

Kallio, E. I., Pulkkinen, T. I., Koskinen, H. E. J., Viljanen, A., Slavin, J. A., and Ogilvie, K.: Loading-unloading process in the nightside ionosphere, Geophys. Res. Lett., 27, 1627-1630, 2000.

Kubyshkina, M., Pulkkinen, T. I., Ganushkina, N., and Partamies, N.: Magnetospheric currents during sawtooth events: Eventoriented magnetic field model analysis, J. Geophys. Res., 113, A08211, doi:10.1029/2007JA012983, 2008.

Kullen, A. and Karlsson, T.: On the relation between solar wind, pseudobreakups, and substorms, J. Geophys. Res., 109, A12218, doi:10.1029/2004JA010488, 2004.

McPherron, R. L.: Growth phase of magnetospheric substorms, J. Geophys. Res., 75, 5592-5599, 1970.

McPherron, R. L.: Magnetospheric substorms, Rev. Geophys. Space Phys., 17, 657-681, 1979.

McPherron, R. L. and Hsu, T.-S.: A comparison of substorms occurring during magnetic storms with those occurring during quiet times, J. Geophys. Res., 107, 1259, doi:10.1029/2001JA002008, 2002.

O'Brien, T. P., Thompson, S. M., and McPherron, R. L.: Steady magnetospheric convection: Statistical signatures in the solar wind and AE, Geophys. Res. Lett., 29, 1130, doi:10.1029/2001GL014641, 2002.

Partamies, N., Pulkkinen, T. I., McPherron, R. L., McWilliams, K., Bryant, C. R., Tanskanen, E., Singer, H. J., Reeves, G. D., and Thomsen, M. F.: Different magnetospheric modes: solar wind driving and coupling efficiency, Ann. Geophys., 27, 4281-4291, doi:10.5194/angeo-27-4281-2009, 2009.

Partamies, N., Juusola, L., Tanskanen, E., Kauristie, K., Weygand, J. M., and Ogawa, Y.: Substorms during different storm phases, Ann. Geophys., 29, 2031-2043, doi:10.5194/angeo-292031-2011, 2011. 
Pulkkinen, T. I., Partamies, N., McPherron, R. L., Henderson, M., Reeves, G. D., Thomsen, M. F., and Singer, H.: Statistical analysis of stormtime activations and sawtooth events, J. Geophys. Res., 112, A01205, doi:10.1029/2006JA012024, 2007.

Rostoker, G., Akasofu, S.-I., Foster, J., Greenwald, R., Kamide, Y., Kawasaki, K., Lui, A., McPherron, R., and Russell, C.: Magnetospheric substorms - Definitions and signatures, J. Geophys. Res., 85, 1663-1668, doi:10.1029/JA085iA04p01663, 1980.

Rostoker, G., Akasofu, S.-I., Baumjohann, W., Kamide, Y., and McPherron, R. L.: The roles of direct input of energy from the solar wind and unloading of stored magnetotail energy in driving magnetospheric substorms, Space Sci. Rev., 46, 93-111, 1987.

Sergeev, V. A., Pellinen, R. J., and Pulkkinen, T. I.: Steady magnetospheric convection: a review of recent results, Space Sci. Rev., 75, 551-604, 1996.
Tanskanen, E.: A comprehensive high-throughput analysis of substorms observed by IMAGE magnetometer network: Years 1993-2003, J. Geophys. Res., 114, A05204, doi:10.1029/2008JA013682, 2009.

Tanskanen, E., Pulkkinen, T. I., Koskinen, H. E. I., and Slavin, J. A.: Substorm energy budget during low and high solar activity: 1997 and 1999 compared, J. Geophys. Res., 107, 1086, doi:10.1029/2001JA900153, 2002.

Tanskanen, E. I., Slavin, J. A., Tanskanen, A. J., Viljanen, A., Pulkkinen, T. I., Koskinen, H. E. J., Pulkkinen, A., and Eastwood, J.: Magnetospheric substorms are strongly modulated by interplanetary high-speed streams, Geophys. Res. Lett., 32, L16104, doi:10.1029/2005GL023318, 2005. 